\title{
Direct observation of the site-specific valence electronic structure at $\mathrm{SiO}_{2} / \mathrm{Si}(111)$ interface
}

\author{
Y. Yamashita, ${ }^{*}$ S. Yamamoto ${ }^{\dagger}$ K. Mukai, and J. Yoshinobu \\ The Institute for Solid State Physics, The University of Tokyo, Kashiwa, Chiba 277-8581, Japan \\ Y. Harada, T. Tokushima, T. Takeuchi, and Y. Takata \\ Riken/SPring-8, Sayo-gun, Hyogo 679-5148, Japan \\ S. Shin \\ The Institute for Solid State Physics, The University of Tokyo, Kashiwa, Chiba 277-8581, Japan and \\ Riken/SPring-8, Sayo-gun, Hyogo 679-5148, Japan
}

K. Akagi and S. Tsuneyuki

Department of Physics, Graduate School of Science,

The University of Tokyo, Bunkyo-ku, Tokyo 113-0033, Japan

(Received 17 January 2006; Accepted 15 February 2006; Published 24 March 2006)

\begin{abstract}
For today's silicon based devices, understanding the $\mathrm{SiO}_{2} / \mathrm{Si}(111)$ interface on atomic level is an important subject for fabricating superior devices. However, despite of many studies on the $\mathrm{SiO}_{2} / \mathrm{Si}(111)$ interface, the interfacial valence electronic states have been typically evaluated as the average and not as individual states. In the present study, we successfully observed valence electronic states of particular atoms at the $\mathrm{SiO}_{2} / \mathrm{Si}(111)$ interface for the first time using soft x-ray absorption and emission spectroscopy. In addition, comparing the experimental results to first-principles calculations revealed local interfacial properties. [DOI: 10.1380/ejssnt.2006.280]
\end{abstract}

Keywords: silicon; silicon oxides; semiconductor-insulator interfaces; X-ray emission; density functional calculations; interface

\section{INTRODUCTION}

Over the last 30 years, the active element density in integrated circuits has doubled roughly every 18 months as embodied in Moore's law. The smallest, but crucial, controlling feature of these circuits is the gate oxide (amorphous $\mathrm{SiO}_{2}$ ) layer, which is the basis for metaloxide-semiconductor field effect transistors (MOSFET's). Currently, industry can produce MOSFET's with a gate shorter than $50 \mathrm{~nm}$ and an $\mathrm{SiO}_{2}$ gate oxide less than $2 \mathrm{~nm}$ thick [1]. If the present reductions continue, the thickness will soon be a few atomic layers. This implies that an atomic level understanding of the properties at the $\mathrm{SiO}_{2} / \mathrm{Si}$ interface is important for fabricating devices with an ultra-thin oxide layer. Therefore, we have focused on measuring the occupied electronic states projected at particular atoms of the $\mathrm{SiO}_{2} / \mathrm{Si}(111)$ interface.

Recently, several groups reported that the conduction band edge was lower at the interface compared to bulk $\mathrm{SiO}_{2}$ [2-4]: Muller, et al. reported that the oxygen $K$ edge was $3 \mathrm{eV}$ lower at the interface using electron energy loss spectroscopy (EELS) in a scanning transmission microscope [2]. Based on this finding, the selective photoabsorption at the interface is realized by tuning the incident photon energy to only allow the electronic excitation from the $\mathrm{O} 1 \mathrm{~s}$ level to the lowered conduction band levels. Once the $\mathrm{O} 1 s$ core level at the interface is excited, the core hole is filled by an electron from an occupied valence level accompanied with soft X-ray emission. Since the O $1 s$ state is localized on a particular oxygen site, the emit-

\footnotetext{
*Corresponding author: yyama@issp.u-tokyo.ac.jp

${ }^{\dagger}$ Present address: Stanford Synchrotron Radiation Laboratory, 2575 Sand Hill Road, Menlo Park, CA 94025, USA
}

ted photon spectrum allows an atom-specific projection of the valence electronic states. Using this method, we successfully observed site-specific occupied states at the $\mathrm{SiO}_{2} / \mathrm{Si}(111)$ interface for the first time.

\section{EXPERIMENTAL}

$\mathrm{SiO}_{2} / \mathrm{Si}(111)$ samples were prepared from phosphorusdoped $n$-type $\mathrm{Si}(111)$ wafers that have a resistivity of $0.001 \Omega \mathrm{cm}$. After standard RCA cleaning, a native oxide layer was etched away by a $1 \% \mathrm{HF}$ solution and then the wafers were immersed in a $40 \% \mathrm{NH}_{4} \mathrm{~F}$ solution for $15 \mathrm{~min}$ to form an atomically smooth silicon surface $[\mathrm{Si}(111)(1 \times 1)-\mathrm{H}][5,6]$. A $1.8 \mathrm{~nm}$-oxide-layer was prepared in $0.1 \mathrm{M} \mathrm{Pa}$ of oxygen at $600 \mathrm{~K}$ for $5 \mathrm{~min}$.

The synchrotron radiation experiments were performed using BL-27SU at SPring-8. The oxygen $K$-edge absorption spectroscopy was performed by detecting $\mathrm{O} \mathrm{KVV}$ Auger electrons at $510 \mathrm{eV}$ and an instrumental energy resolution of $50 \mathrm{meV}$. Soft X-ray emission (SXE) spectra were measured with an $800 \mathrm{meV}$ energy resolution. The design and performance of the SXE spectrometer is described in detail elsewhere [7]. Si $2 p$ core level spectra were measured with incident photon energy of $800 \mathrm{eV}$ and a Gammadata Scienta SES2002.

Atomic force microscopy (AFM) measurements were performed with a JEOL SPM 4200 using contact mode. The H-terminated $\mathrm{Si}(111)$ surface and the surface after the etch of the oxide layer of $\mathrm{SiO}_{2}$ on $\mathrm{Si}(111)$ away with a $0.5 \% \mathrm{HF}$ solution were investigated. The measurements were performed in nitrogen atmosphere.

First-principles calculations based on the density functional theory with a generalized gradient approximated correction were performed to evaluate the local density of states of the valence level and the interface structure 

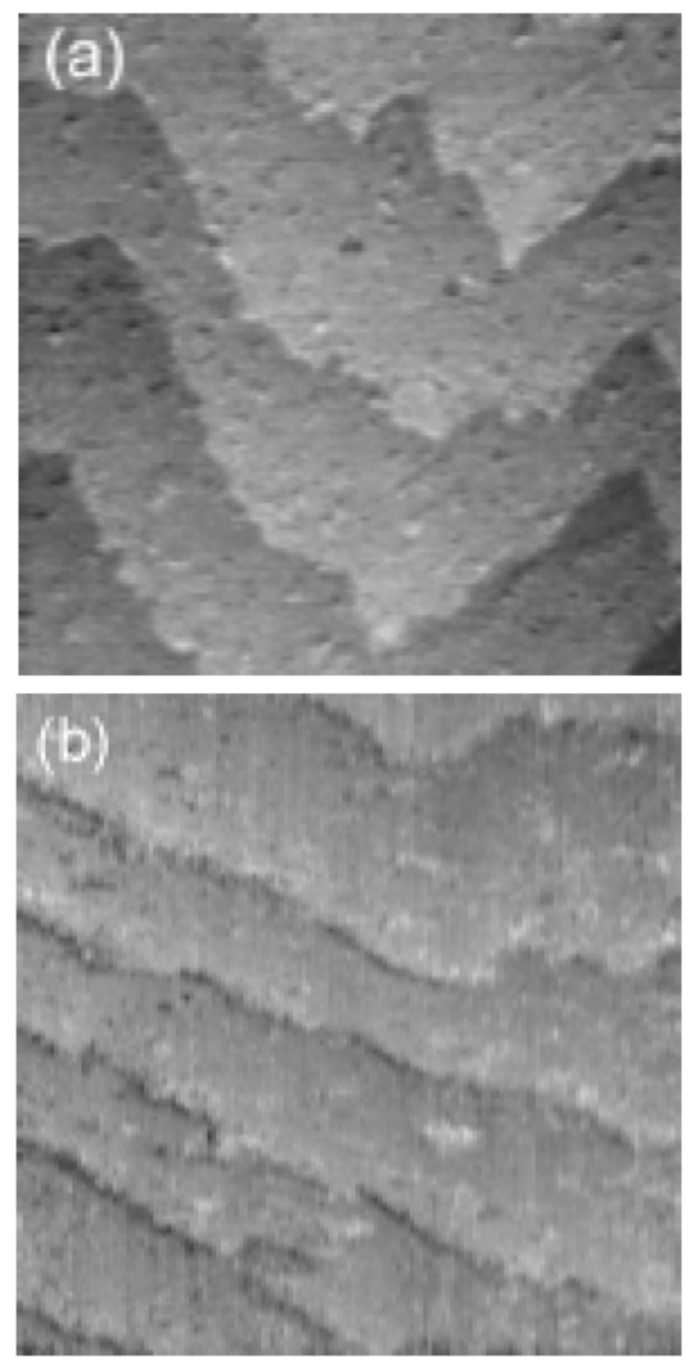

FIG. 1: AFM images: (a) the H-terminated Si(111) surface and (b) after the etch of the oxide layer on $\mathrm{Si}(111)$ away. Each area is $300 \times 300 \mathrm{~nm}^{2}$ and the measurements were performed in nitrogen atmosphere.

and properties [8]. A six Si-layer thick slab model was used with a periodic boundary condition: the unit cell contains four dangling bonds of a clean $\mathrm{Si}(111)$ surface in its initial configuration. The oxidized structures were then obtained by inserting $\mathrm{O}$ atoms one by one with structure relaxation. We prepared three models obtained by a different insertion order of oxygen atoms.

\section{RESULTS AND DISCUSSION}

Figure 1 shows AFM images of the H-terminated Si(111) surface and after the etch of the oxide layer on $\mathrm{Si}(111)$ away. After the etch of the oxide layer, steps are clearly observed indicating that the interface is atomically flat after the oxidation. This is consistent with the previous studies $[6,9]$.

Figures 2(a) and (b) show the oxygen $K$-edge absorption spectra of $\mathrm{SiO}_{2} / \mathrm{Si}(111)$ structures with $1.8-\mathrm{nm}$ and 8-nm thick $\mathrm{SiO}_{2}$ layers, respectively. The latter is considered to represent the bulk $\mathrm{SiO}_{2}$ spectrum since the mean

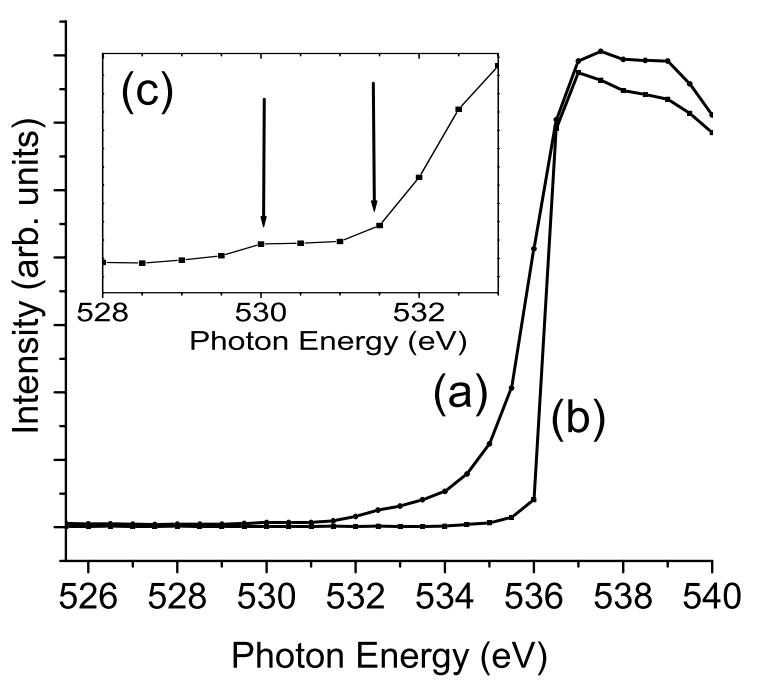

FIG. 2: Oxygen $K$-edge absorption spectra of (a) 1.8-nm-thick $\mathrm{SiO}_{2} / \mathrm{Si}(111)$ and (b) 8-nm-thick $\mathrm{SiO}_{2} / \mathrm{Si}(111)$ structures. The spectrum (c) shows the magnified spectrum of (a). Incident photon angle was $36^{\circ}$ from surface normal and $p$-polarized photon was used.

free path of the Auger electron is $\sim 1.5 \mathrm{~nm}$ at $510 \mathrm{eV}$ [10], which is much shorter than the $\mathrm{SiO}_{2}$ layer thickness. An absorption spectrum of the 1.8-nm-thick $\mathrm{SiO}_{2} / \mathrm{Si}(111)$ structure is strikingly different from the bulk $\mathrm{SiO}_{2}$ spectrum. The $1.8 \mathrm{~nm}$ spectrum has a lower onset and exhibits edge structures at 530 and $531.5 \mathrm{eV}$. Muller et al. used EELS and reported that the oxygen $K$-edge was lowered by $3 \mathrm{eV}$ at the interface compared to the bulk $\mathrm{SiO}_{2}$ [2]. Thus, the edge structures in the spectrum are explained by unoccupied $\mathrm{O} 2 p$ states at the interface, and the lowered edge exhibits a reduced band gap at the interface. Note that the peak at $537.5 \mathrm{eV}$ is attributed to unoccupied O $2 p$ states bound with $\mathrm{Si} 3 s$ and $3 p$ states of bulk $\mathrm{SiO}_{2}[11,12]$.

Figure 3 shows the Si $2 p$ core level spectrum for the 1.8-nm-thick $\mathrm{SiO}_{2} / \mathrm{Si}(111)$ structure. A peak fitting procedure was referred to the previous studies $[5,6,13]$. In these figures, $\mathrm{Si}^{0}$ and $\mathrm{Si}^{4+}$ denote the $\mathrm{Si}$ substrate and $\mathrm{SiO}_{2}$ respectively, and $\mathrm{Si}^{1+}$ and $\mathrm{Si}^{3+}$ denote $\mathrm{Si}$ atoms bound to one and three oxygen atoms, respectively. Note that $\mathrm{Si}^{2+}$ due to an $\mathrm{Si}$ atom bound to two oxygen atoms was very weak for the 1.8 -nm-thick $\mathrm{SiO}_{2} / \mathrm{Si}(111)$ structure. It is found from emission angle dependence of Si $2 p$ spectra that these suboxide species, i.e. $\mathrm{Si}^{1+}$, $\mathrm{Si}^{2+}$, and $\mathrm{Si}^{3+}$, were dominantly formed at the interface. In addition, analysis of the Si $2 p$ photoelectron spectra for the 1.8-nm-thick $\mathrm{SiO}_{2} / \mathrm{Si}(111)$ structure revealed that the relative intensities of the suboxide species were $S i^{1+}: S i^{2+}: S i^{3+}=0.563: 0.003: 0.434$. Thus the interface predominantly consists of $\mathrm{Si}^{1+}$ and $\mathrm{Si}^{3+}$ and these results are good agreement with the previous results [5].

Wallis, et al. reported using theoretical calculations that the oxygen $K$-edge of the intermediate states in the amorphous silicon shifted to lower energy as the oxidation number of the adjacent silicon atoms decreased [3]. 

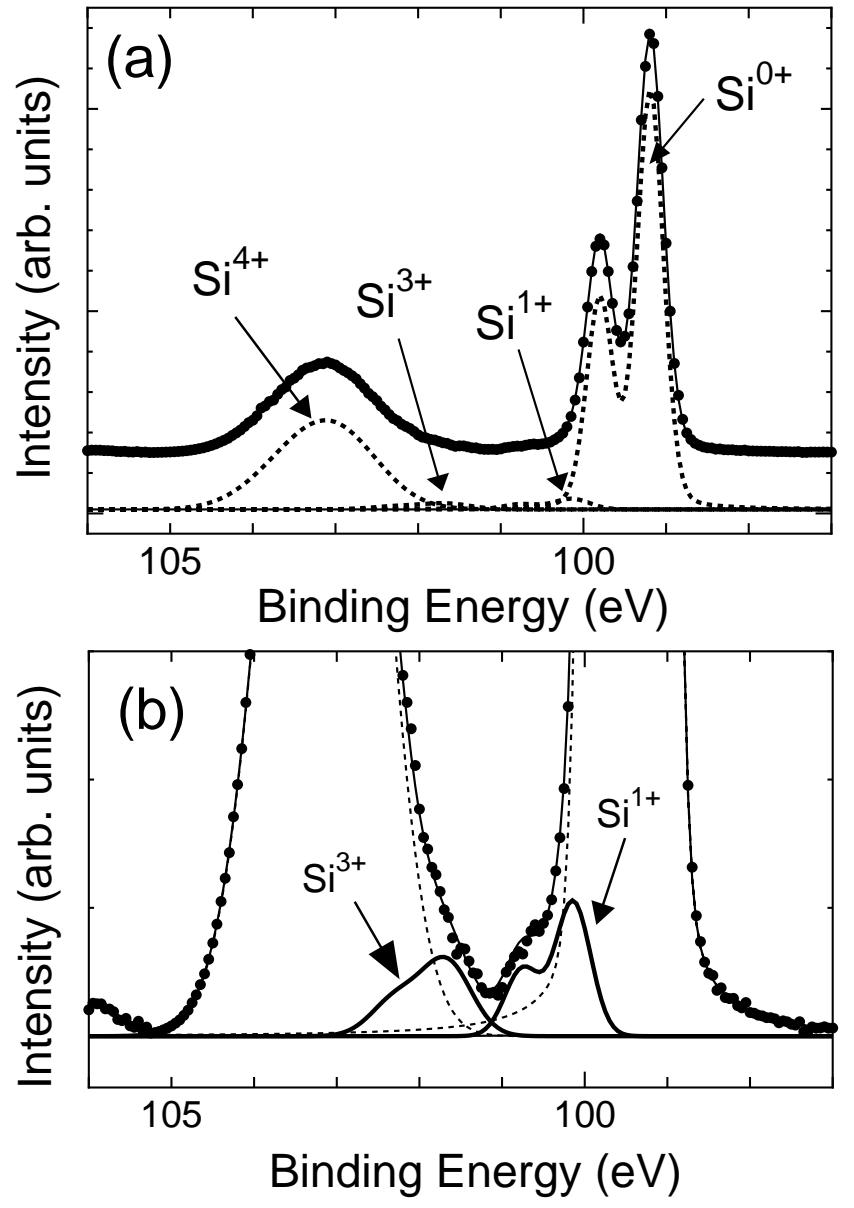

FIG. 3: Si $2 p$ core level spectrum for (a) 1.8-nm-thick $\mathrm{SiO}_{2} / \mathrm{Si}(111)$ and (b) the magnified spectrum of (a). The spectrum was measured at the photon energy of $800 \mathrm{eV}$ and take-off angle of $45^{\circ}$.

Thus, the absorption edges at 530 and $531.5 \mathrm{eV}$ are assigned to an $\mathrm{O}$ atom bonding to $\mathrm{Si}^{1+}$ and $\mathrm{Si}^{3+}$ at the interface, respectively (hereafter, the $\mathrm{O}$ atom bonding to $\mathrm{Si}^{1+}$ and $\mathrm{Si}^{3+}$ at the interface is denoted to $\mathrm{P} 1$ and $\mathrm{P} 3$, respectively). Therefore, site-specific SXE spectra of particular oxygen atoms at the interface can be obtained by choosing excitation energies, i.e., $530 \mathrm{eV}$ for P1, $531.5 \mathrm{eV}$ for P3, and $537.5 \mathrm{eV}$ for $\mathrm{SiO}_{2}$.

Figures 4(a)-(c) show the O $K$-edge SXE spectra for the 1.8-nm-thick $\mathrm{SiO}_{2} / \mathrm{Si}(111)$ structure. The electronic states at the interface are noticeably different from those of bulk $\mathrm{SiO}_{2}$ and the interfacial electronic states strongly depend on the intermediate oxidation states at the interface. With a photon energy of $537.5 \mathrm{eV}$, the occupied $\mathrm{O}$ $2 p$ states of bulk $\mathrm{SiO}_{2}$ predominantly contribute to the SXE spectrum (Fig. 4(a)). In this spectrum, the peak at $7.25 \mathrm{eV}$ and the shoulder at $8.65 \mathrm{eV}$ are attributed to the O $2 p$ non-bonding states, while the peaks at 11.75 and $14.33 \mathrm{eV}$ are due to the bonding states between $\mathrm{O} 2 p$ and $\mathrm{Si} s p^{3}$ orbitals [14]. When the incident photon energy is $533 \mathrm{eV}$ in order to excite P3 (Fig. 4(b)), the peaks due to non-bonding states become broader and shift towards a higher binding energy compared to bulk $\mathrm{SiO}_{2}$. In addition, a new, small, broad peak around $4 \mathrm{eV}$ is observed. For P1 (Fig 4. (c)), the peak at $6.50 \mathrm{eV}$, which is at-

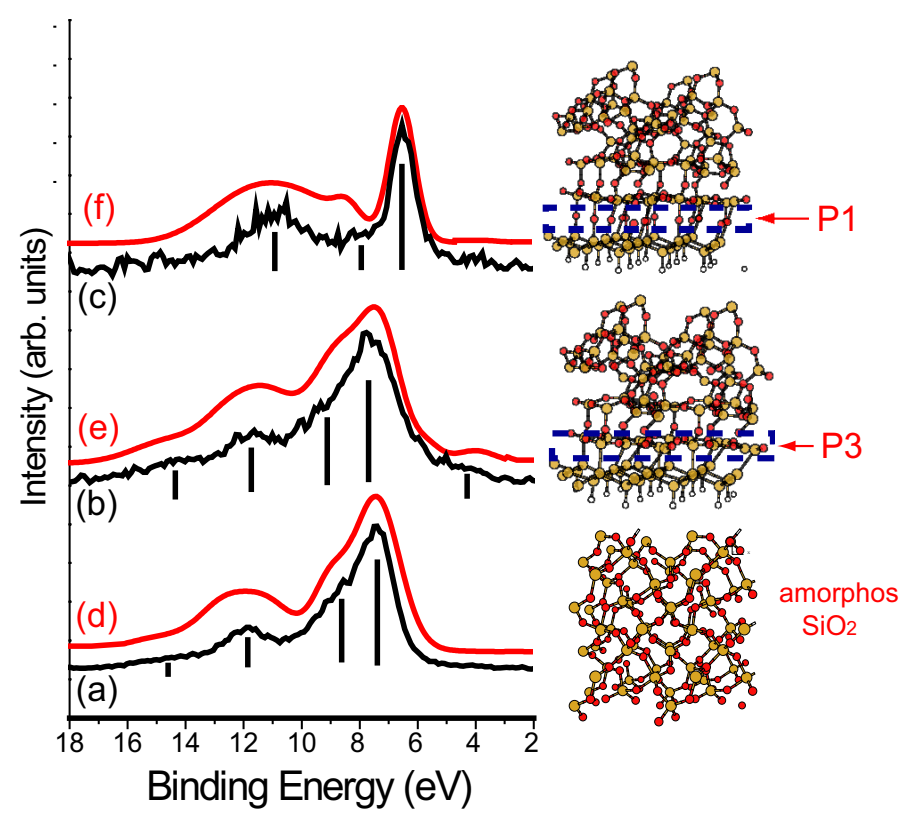

FIG. 4: O $K$-edge SXE spectra for the 1.8-nm-thick $\mathrm{SiO}_{2} / \mathrm{Si}(111)$ structure and the calculated O- $2 p$ density of states (DOS) obtained from the model presented in this figure. For the SXE spectra shown as the black lines, incident photon energies were (a) $537.5 \mathrm{eV}$, (b) $533 \mathrm{eV}$, and (c) 530 $\mathrm{eV}$. Incident photon angle was $60^{\circ}$ from surface normal and a $p$-polarized photon was used. Note that in order to selectively excite P3, we used $533 \mathrm{eV}$ as the incident photon energy. Red lines show the calculated O- $2 p$ DOS for (d) $\mathrm{SiO}_{2}$, (e) P3 atoms of $\mathrm{SiO}_{2} / \mathrm{Si}(111)$ interface with $\mathrm{P} 3$, and (f) $\mathrm{P} 1$ atoms of $\mathrm{SiO}_{2} / \mathrm{Si}(111)$ interface with $\mathrm{P} 1$. The calculated DOS spectrum was the average of each oxygen atom spectrum for amorphous $\mathrm{SiO}_{2}$ and that of each interface oxygen atom spectrum in the case of P1 and P3. Gaussian broadening was applied for the O- $2 p$ DOS using experimental and lifetime broadening (total $1.1 \mathrm{eV}$ ). Red balls and yellow balls correspond to oxygen and silicon atoms, respectively.

tributed to the non-bonding states of $\mathrm{O} 2 p$, is very sharp and does not have a shoulder, indicating that an uniform structure is formed at the interface. Note that P1 should be also excited as well as P3 when the incident photon energy is $533 \mathrm{eV}$ because the absorption edge of P3, i.e. $531.5 \mathrm{eV}$, could overlap with that of P1 (see Fig. 2(c)). However, intensity of $\mathrm{P} 1$ decreased as increasing incident photon energy; the relative intensity of $\mathrm{P} 1$ was less than $10 \%$ judging from the $\mathrm{P} 1$ derived peak intensity at 6.50 $\mathrm{eV}$. Thus, the occupied O $2 p$ states of P3 predominantly contribute to the SXE spectrum (Fig. 4(b)) when the incident photon energy is $533 \mathrm{eV}$.

A straightforward way to quantitatively evaluate the interface states is to combine the experimental results with theoretical calculations, providing the interface properties such as the structure, conducting and dielectric behavior. For the $\mathrm{SiO}_{2} / \mathrm{Si}(111)$ interface, the interface is abrupt, and $\mathrm{Si}^{1+}$ and $\mathrm{Si}^{3+}$ species dominate as described above. Therefore, the atomically flat $\mathrm{SiO}_{2} / \mathrm{Si}(111)$ interface only consisting of $\mathrm{Si}^{1+}$ or $\mathrm{Si}^{3+}$ is calculated as the interface model. Figures 4(d)-(f) show the oxygen projected density of states $\left(\mathrm{O}-2 p\right.$ DOS) for $\mathrm{SiO}_{2}, \mathrm{P} 3$, and $\mathrm{P} 1$, respec- 


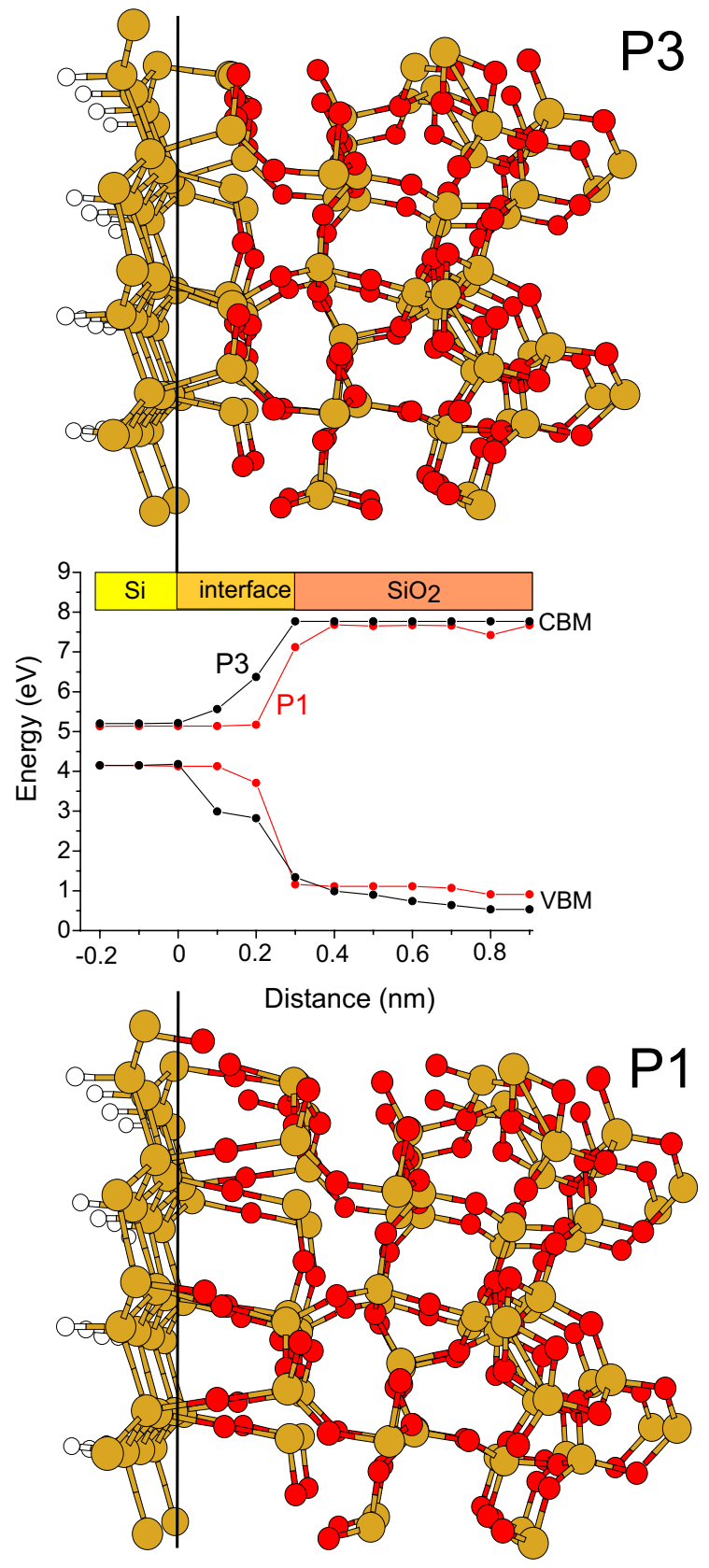

FIG. 5: Calculated energy band gap variation in the structures with $\mathrm{P} 1$ and $\mathrm{P} 3$ along the normal direction to the interface. Black and red solid lines exhibit the structure with P3 and P1 interfaces, respectively. The solid lines in the interface model structures show the origin of the distance.

tively, calculated with the model structures illustrated in the right.

It is clear that the calculated DOS for amorphous $\mathrm{SiO}_{2}$ produces SXE spectrum of the oxide region well (Figs. $4(\mathrm{a})(\mathrm{d}))$. The Si-O-Si angle and Si-O bond length in the theoretically optimized structure are predominantly distributed from $140^{\circ}$ to $155^{\circ}$ and $0.155 \mathrm{~nm}$ to $0.165 \mathrm{~nm}$, respectively, which are consistent with previous experimental results [15]. As for the P3 interface layer, the $\mathrm{Si}-\mathrm{O}-\mathrm{Si}$ angle is distributed from $131.6^{\circ}$ to $151.6^{\circ}$ and the $\mathrm{Si}-\mathrm{O}$ bond length from $0.158 \mathrm{~nm}$ to $0.176 \mathrm{~nm}$ in the theoretically optimized structure. The local variation from the amorphous $\mathrm{SiO}_{2}$ results in the appearance of a shoul- der at $4 \mathrm{eV}$ and the broadening of the non-bonding state from $7.75 \mathrm{eV}$ to $9.13 \mathrm{eV}$ (Figs. 4(b)(e)). On the other hand, for the P1 interface layer, the Si-O-Si angle and Si$\mathrm{O}$ bond length should be maintained near $180^{\circ}$ and 0.19 $\mathrm{nm}$, respectively, in order to reproduce the non-bonding sharp peak at $6.50 \mathrm{eV}$ (Figs. 4(c)(f)).

Next we investigated how the structure with P1 or P3 interface exhibits the interface properties, which would enable us to understand atomic scale transport properties of carrier. Figure 5 shows the calculated conduction band minimum (CBM) and valence band maximum (VBM) of the structure with $\mathrm{P} 1$ or P3 interface in the interfacial region. For the $\mathrm{P} 1$ interface, since the $\mathrm{Si}-\mathrm{O}-\mathrm{Si}$ bond angle and the $\mathrm{Si}-\mathrm{O}$ bond length are near $180^{\circ}$ and $0.19 \mathrm{~nm}$, the orbital of the non-bonding state is along the parallel to the interface. In this case, our calculations clearly show that the non-bonding state of P1 hardly interacts with $\mathrm{Si}$ atoms, and the CBM and VBM in the interface region are attributed to penetration from DOS of the silicon substrate. Therefore the P1 interface exhibits a similar band gap to the bulk $\mathrm{Si}$ in the interfacial region from $0 \mathrm{~nm}$ to $0.2 \mathrm{~nm}$. For the P3 interface, on the other hand, the non-bonding state of the P3 interacts with Si atoms because the $\mathrm{Si}-\mathrm{O}-\mathrm{Si}$ bond angle and the $\mathrm{Si}-\mathrm{O}$ bond length are distributed as described above. Thus the gap of the P3 interface gradually becomes large in the interfacial region from $0 \mathrm{~nm}$ to $0.2 \mathrm{~nm}$. These results indicate that the P1 interface is relatively more conductive. Thus, the interface characteristics strongly depend on the local interface structures, which implies that performance of MOSFET with an extremely small gate width is essentially affected by the suboxide composition at the interface. In future MOSFET, it is determined that controlling the interfacial states on atomic level is crucial for not only $\mathrm{SiO}_{2}$ less $1 \mathrm{~nm}$ thick, but high-dielectric constant materials and silicon oxynitride used as alternatives to $\mathrm{SiO}_{2}[16,17]$. In this study, we present our approach using SXE, which successfully detects the electronic states of particular atoms at the interface. Therefore, this approach is applicable to interfacial states for various kinds of gate oxides and will be indispensable for evaluating and designing nm-scale superior devices.

\section{CONCLUSIONS}

Site-specific valence electronic states at the $\mathrm{SiO}_{2} / \mathrm{Si}(111)$ interface were successfully observed by SXA and SXE spectroscopy. We found that the interfacial valence electronic states were noticeably different from those of bulk $\mathrm{SiO}_{2}$ and strongly depended on the chemical states of each atom at the interface. In addition, comparison of the experimental results with first-principles calculations quantitatively revealed not only the interface structure but also the interface electronic properties in atomic-scale.

\section{Acknowledgments}

We wish to thank Professors Hattori and Nohira for their useful discussion and gratefully acknowledge the help by Dr. Tamenori of JASRI. This work was supported 
by the Nanotechnology Support Project of the Ministry of Education, Culture, Sports, Science and Technology. The synchrotron radiation experiments were performed at the SPring- 8 with the approval of JASRI (Proposal No. 2003B0209-Nsa-np-Na and 2004A0345-Nsa-np-Na).
[1] International Technology Roadmap for Semiconductors, 2005 ed. Available from the International Technology Roadmap for Semiconductors web site, http://public.itrs.net.

[2] D. A. Muller, T. Sorsch, F. H. Baumann, K. EvansLutterodt, and G. Timp, Nature 399, 758 (1999).

[3] D. J. Wallis, P.H. Gaskell, and R. Brydson, J. Microsc. 180, 307 (1995)

[4] J. B. Neaton, D. A. Muller, and N. W. Ashcroft, Phys. Rev. Lett. 85, 1298 (2000).

[5] K. Ohishi and T. Hattori, Jpn. J. Appl. Phys. 33, L675 (1994).

[6] Y. Yamashita, A. Asano, Y. Nishioka, and H. Kobayashi, Phys. Rev. B 59, 15872 (1999).

[7] T. Tokushima, Y. Harada, M. Watanabe, Y. Takata, E. Ishiguro, A. Hiraya, and S. Shin, Sur. Rev. Lett. 9, 503 (2002).

[8] J. Yamauchi, M. Tsukada, S. Watanabe, and O. Sugino, Phys. Rev. B 54, 5586 (1996).

[9] T. Komeda, K. Namba, and Y. Nishioka, Jpn. J. Appl. Phys. 37, L214 (1998).
[10] The electron inelastic-mean-free-paths were estimated using NIST Standard Reference Database 71, NIST Electron Inelastic-Mean-Free-Path Database, Ver. 1.1.

[11] Z. Y. Wu, F. Jollet, and F. Seifer, J. Phys.:Condens. Matter 10, 8083 (1998).

[12] J. Sarnthein, A. Pasquarello, and R. Car, Phys. Rev. Lett. 74, 4682 (1995).

[13] Y. Yamashita, S. Machida, M. Nagao, S. Yamamoto, Y. Kakefuda, K. Mukai, and J. Yoshinobu, Jpn. J. Appl. Phys. 41, L272 (2002).

[14] G. Hollinger, E. Bergigna, H. Chermette, F. Himpsel, D. Lohex, M. Lannoo, and M. Bensoussan, Philos. Mag. B 55, 735 (1987).

[15] E. Dupree and R. F. Pettifer. Nature 308, 523 (1984).

[16] K. Inoue, K. Furuno, H. Kato, N. Tamura, K. Hikazutani, S. Sano and T. Hattori, Jpn. J. Appl. Phys. 40, L539 (2001).

[17] G. D. Wilk, R.M Wallace, and J.M. Anthony, J. Appl. Phys 89, 5243 (2001). 